\title{
A NONLINEAR SINGULAR PERTURBATION PROBLEM ON A SEMI-INFINITE INTERVAL
}

\section{J. J. SHEPHERD}

(Received 28 September 1977)

(Revised 21 November 1977)

\begin{abstract}
We consider a nonlinear singular perturbation problem on a semi-infinite interval, that is a generalization of the well-known Lagerstrom model equation intended to model low Reynolds number flow. By applying a Green's function method and the contraction mapping principle, we are able to obtain existence, uniqueness and asymptoticity results for this problem.
\end{abstract}

\section{Introduction}

In this article, we consider the solutions $y(x, \varepsilon)$ of the nonlinear two-point boundary-value problem $P_{\epsilon}$, defined by the differential equation

$$
y^{\prime \prime}+\frac{k}{x} y^{\prime}+\varepsilon f(y, x) y^{\prime}=0, \quad x \in(1, \infty),
$$

and the boundary conditions

$$
\begin{gathered}
y(1, \varepsilon)=a, \\
y(\infty, \varepsilon)=1,
\end{gathered}
$$

where $\varepsilon$ is a small real positive parameter, with $\varepsilon \in\left(0, \varepsilon_{0}\right], k$ is a positive integer, and $a$ is a constant, independent of $\varepsilon$, with $a \geqslant 0$.

The problem $P_{\varepsilon}$ is of interest because of the weak nonlinearity involved, and also because, for certain choices of $k, f$ and $a$, it is a singular perturbation problem, in the sense that there is no asymptotic expansion in $\varepsilon$ of Poincaré type that approximates $y(x, \varepsilon)$ uniformly on the interval $[1, \infty)$. In fact, attempts to construct such an expansion lead to insoluble boundary-value problems. This is most striking 
in the case $k=1$, where the reduced problem $P_{0}$, obtained by setting $\varepsilon=0$ in $P_{\epsilon}$, has no solution that satisfies both the boundary conditions (1.2) and (1.3), for arbitrary values of $a$.

For arbitrary $k$ and $f(y, x)=y, a=0$, the problem $P_{\varepsilon}$ becomes the well-known Lagerstrom model problem (Lagerstrom [5]), which is intended to model the phenomenon of low Reynolds number flow in the region exterior to a finite body, the boundary of this body being represented by $x=1$. Here, the parameter $\varepsilon$ plays the role of the Reynolds number, and the boundary condition (1.3) corresponds to the condition of uniform streaming at infinity. This model problem is well represented in the literature (Bush [2], Cole [3], Hsiao [4], Lagerstrom and Casten [6]), where it is used to illustrate the so-called method of matched asymptotic expansions. It seems a reasonable assumption that, under suitable regularity hypotheses about $f(y, x)$, the more general problem $P_{\varepsilon}$ might be used in a similar way. However, it is not our aim in this paper to apply such methods to $P_{6}$, although we will be concerned with the construction of suitable approximations to the solutions of the problem. What is of much greater relevance to our discussion is the material of a recent paper (Rosenblat and Shepherd [9]), in which the authors establish existence, uniqueness and asymptoticity properties (as $\varepsilon \rightarrow 0$ ) for the solution of the Lagerstrom model problem, by comparing this (nonlinear) problem with a suitably chosen linear problem. Thus in the present paper, we will apply this technique to the problem $P_{\varepsilon}$, to obtain similar properties, under suitable assumptions about the function $f(y, x)$.

The physical analogy between the Lagerstrom model problem and the equations of fluid motion at low Reynolds numbers has led to the proposal [5] that a suitable first approximation to the solution of this problem might be obtained from the solution of a model Oseen equation, in which the nonlinear term in the differential equation is replaced by one linearized about $y=1$. This is the particular linear problem used in [9]. Prompted by this linearization, we generalize it, and propose that the function $Y(x, \varepsilon)$, defined to be the solution of the linear problem $L_{\epsilon}$, given by

$$
\begin{aligned}
Y^{\prime \prime}+\frac{k}{x} Y^{\prime}+\varepsilon f(1, x) Y^{\prime} & =0, \quad x \in(1, \infty), \\
Y(1, \varepsilon) & =a, \\
Y(\infty, \varepsilon) & =1,
\end{aligned}
$$

will, under conditions to be determined, provide a suitable first approximation to the solution $y(x, \varepsilon)$ of $P_{e}$. If the equation (1.1) is viewed as a generalized low Reynolds number flow equation, the equation (1.4) should be viewed as a generalized Oseen equation for the same type of flow. 
In the following sections, we will show that the problems $P_{\epsilon}$ and $L_{\epsilon}$ have solutions $y(x, \varepsilon)$ and $Y(x, \varepsilon)$ respectively, with $y(x, \varepsilon)$ tending to the value 1 as $x \rightarrow \infty$, in the sense that

$$
\varepsilon \int_{1}^{\infty}|1-y(s, \varepsilon)| d s=O(1)
$$

as $\varepsilon \rightarrow 0$; and further, that the solution $Y(x, \varepsilon)$ is, in an appropriate sense, a uniform asymptotic approximation to $y(x, \varepsilon)$. Moreover, we will demonstrate that $y(x, \varepsilon)$ has a uniformly valid generalized asymptotic expansion of the form

$$
y(x, \varepsilon)=Y(x, \varepsilon)+\sum_{i=1}^{N} \nu_{i}(\varepsilon) Y_{i}(x, \varepsilon)+O\left(\nu_{N+1}(\varepsilon)\right),
$$

where $\left\{\nu_{i}(\varepsilon)\right\}_{i=1}^{\infty}$ is an asymptotic sequence, $\left\{Y_{i}(x, \varepsilon)\right\}_{i=1}^{\infty}$ is a sequence of functions that are $O(1)$ as $\varepsilon \rightarrow 0$ for all $x \in[1, \infty)$; and we will obtain an algorithm for the construction of this expansion. This algorithm, which involves only systematic integration, and which requires only relatively mild restrictions on the function $f(y, x)$ will be seen to have distinct advantages over the usual series type of construction, that would usually require $f(y, x)$ to be analytic in the argument $y$.

\section{Assumptions and preliminary estimates}

The discussion of the previous section shows clearly the important part played by the linear problem $L_{\varepsilon}$ defined by (1.4)-(1.6). Thus, it is important to examine the general features of its solution $Y(x, \varepsilon)$. Obviously, some restriction must be imposed on the behaviour of $f(1, x)$; and the following assumption suffices for the purpose of this paper:

( $F) f(1, x)$ is continuous and uniformly bounded on the interval $[1, \infty)$ and satisfies the inequality

$$
f(1, x) \geqslant C x^{\alpha-1}
$$

as $x \rightarrow \infty$, where $C, \alpha$ are positive constants independent of $\varepsilon$, with $0<\alpha \leqslant 1$.

LeMmA 1. Let $f(1, x)$ satisfy condition $(F)$. Then, for each $\varepsilon \in\left(0, \varepsilon_{0}\right]$, the linear problem $L_{\epsilon}$ has a solution $Y(x, \varepsilon)$ that may be written in the form

where

$$
Y(x, \varepsilon)=1+(a-1) W(x, \varepsilon)
$$

and

$$
W(x, \varepsilon)=F_{k}(x, \varepsilon) / F_{k}(1, \varepsilon)
$$

$$
F_{k l}(x, \varepsilon)=\int_{x}^{\infty} t^{-k} \exp \left\{-\varepsilon \int_{1}^{t} f(1, s) d s\right\} d t
$$


For each $\varepsilon \in\left(0, \varepsilon_{0}\right]$, we have

while

$$
\min \{a, 1\} \leqslant Y(x, \varepsilon) \leqslant \max \{a, 1\}
$$

$$
\begin{array}{ll}
Y^{\prime}(x, \varepsilon) \geqslant 0 & \text { if } 0 \leqslant a \leqslant 1, \\
Y^{\prime}(x, \varepsilon) \leqslant 0 & \text { if } a \geqslant 1 .
\end{array}
$$

Proof. Equation (1.4) may be written as

$$
\frac{d}{d x}\left\{x^{k} \exp \left(\varepsilon \int_{1}^{x} f(1, s) d s\right) Y^{\prime}\right\}=0
$$

and integration of this, together with the application of the boundary conditions (1.5), (1.6), gives the result (2.2), provided the infinite integrals involved converge. Under the condition $(F)$, there exists a finite $x_{0}$, independent of $\varepsilon$, such that (2.1) holds for all $x \geqslant x_{0}$. Then, for $t \geqslant x_{0}$,

$$
t^{-k} \exp \left\{-\varepsilon \int_{1}^{t} f(1, s) d s\right\} \leqslant \exp \left\{\varepsilon C \alpha^{-1} x_{0}^{\alpha}-\varepsilon \int_{1}^{x_{0}} f(1, s) d s\right\} t^{-k} \exp \left(-\varepsilon C \alpha^{-1} t^{\alpha}\right),
$$

and this establishes convergence of the integrals in $W(x, \varepsilon)$. The results (2.5)-(2.7) then follow, on noting that $0 \leqslant W \leqslant 1$, and $W^{\prime} \leqslant 0$.

REMARK. Note that the assumption (F) is a sufficient condition only. A weaker condition might be

where

$$
f(1, x) \geqslant \phi_{k}(x) \text { as } x \rightarrow \infty \text {, }
$$

$$
\begin{aligned}
& \phi_{1}(x)=C x^{-1} \\
& \phi_{k}(x)=-C x^{-1}, \quad k \geqslant 2,
\end{aligned}
$$

with $C$ a positive constant, independent of $\varepsilon$. In this case, there is no need for the boundedness of $f(1, x)$.

Although the estimates (2.5)-(2.7) give some idea of the behaviour of $Y(x, \varepsilon)$ they give no details of the behaviour of this solution as $\varepsilon \rightarrow 0$, and are insufficiently precise for the purposes of later sections. Such information is provided in the following lemma, which examines the behaviour of the function $W(x, \varepsilon)$ for small values of $\varepsilon$.

LEMMA 2. Let $f(1, x)$ satisfy the condition $(F)$. Then, there exist positive constants $A(\varepsilon), B(\varepsilon)$ that are $O(1)$ and nonvanishing as $\varepsilon \rightarrow 0$, such that

$$
\begin{gathered}
0 \leqslant W(x, \varepsilon) \leqslant A(\varepsilon) V_{k}(x, \varepsilon), \\
B(\varepsilon) V_{k}^{\prime}(x, \varepsilon) \leqslant W^{\prime}(x, \varepsilon) \leqslant 0,
\end{gathered}
$$


uniformly on $[1, \infty)$. Here,

and

$$
V_{k}(x, \varepsilon)=\Gamma_{k}\left(\varepsilon C \alpha^{-1} x^{\alpha}\right) / \Gamma_{k}\left(\varepsilon C \alpha^{-1}\right),
$$

$$
\Gamma_{k}(\lambda)=\int_{\lambda}^{\infty} s^{-k} e^{-s} d s
$$

is an incomplete gamma function, given by

$$
\Gamma_{k}(\lambda)=\lambda^{1-k} E_{k}(\lambda)
$$

where $E_{k}(\lambda)$ is the standard exponential integral $[1, p .229]$.

Proof. We first consider $x \geqslant x_{0}$, where $x_{0}$ is as defined in the proof of Lemma 1 . The first factor on the right-hand side of (2.8) is $O(1)$ and nonvanishing as $\varepsilon \rightarrow 0$. Then, for $x \geqslant x_{0}$,

$$
0 \leqslant F_{k}(x, \varepsilon) \leqslant O(1) \int_{x}^{\infty} t^{-k} \exp \left(-\varepsilon C \alpha^{-1} t^{\alpha}\right) d t,
$$

where the $O(1)$ factor does not vanish as $\varepsilon \rightarrow 0$.

Now set $t^{\alpha}=u$, so that the right-hand side of (2.14) becomes

$$
O(1) \int_{x^{\alpha}}^{\infty} u^{-1-(k-1) \alpha^{-1}} \exp \left(-\varepsilon C \alpha^{-1} u\right) d u .
$$

In (2.15), since $u \geqslant 1$ and $0<\alpha \leqslant 1$, we have, when $k \geqslant 2$,

$$
u^{-1-(k-1) \alpha^{-1}} \leqslant u^{-k}
$$

while the same obviously holds when $k=1$.

Noting that, for any positive $p$ and $q$,

$$
\int_{p}^{\infty} s^{-k} e^{-q s} d s=q^{k-1} \Gamma_{k}(p q)
$$

we have, for $x \geqslant x_{0}$,

$$
F_{k}(x, \varepsilon) \leqslant O(1)\left(\varepsilon C \alpha^{-1}\right)^{k-1} \Gamma_{k}\left(\varepsilon C \alpha^{-1} x^{\alpha}\right) .
$$

Now, letting $G$ be a bound (independent of $\varepsilon$ ) for $|f(1, x)|$ on $[1, \infty$ ), we have

$$
F_{k}(1, \varepsilon) \geqslant e^{e G} \int_{1}^{\infty} t^{-k} e^{-\varepsilon G t} d t,
$$

and thus,

$$
F_{k}(1, \varepsilon) \geqslant e^{e Q}(\varepsilon G)^{k-1} \Gamma_{k}(\varepsilon G) .
$$

The result (2.8) now follows, for $x \geqslant x_{0}$, on applying (2.18) and (2.20), while noting 
that

$$
\Gamma_{k}\left(\varepsilon C \alpha^{-1}\right) / \Gamma_{k}(\varepsilon G)=O(1)
$$

and is nonvanishing as $\varepsilon \rightarrow 0$.

When $x \leqslant x_{0}$, we have

$$
F_{k}(x, \varepsilon) / F_{k}(1, \varepsilon)=F_{k}(1, \varepsilon)^{-1} \int_{x}^{x_{0}} t^{-k} \exp \left\{-\varepsilon \int_{1}^{t} f(1, s) d s\right\} d t+F_{k}\left(x_{0}, \varepsilon\right) / F_{k}(1, \varepsilon) \text {. }
$$

The second term on the right-hand side of (2.22) may be treated by the methods above, to obtain

$$
\begin{aligned}
F_{k}\left(x_{0}, \varepsilon\right) / F_{k}(1, \varepsilon) & \leqslant O(1) \Gamma_{k}\left(\varepsilon C \alpha^{-1} x_{0}^{\alpha}\right) / \Gamma_{k}\left(\varepsilon C \alpha^{-1}\right) \\
& \leqslant O(1) \Gamma_{k}\left(\varepsilon C \alpha^{-1} x^{\alpha}\right) / \Gamma_{k}\left(\varepsilon C \alpha^{-1}\right)
\end{aligned}
$$

where the $O(1)$ factor does not vanish as $\varepsilon \rightarrow 0$.

The first term on the right-hand side of (2.22) may be written

$$
O(1)\left\{\int_{x}^{x_{0}} t^{-k} \exp \left(-\varepsilon \int_{1}^{t} f(1, s) d s\right) d t / \varepsilon^{k-1} \Gamma_{k}\left(\varepsilon C \alpha^{-1} x^{\alpha}\right)\right\} V_{k}(x, \varepsilon)
$$

where the $O(1)$ factor does not vanish as $\varepsilon \rightarrow 0$. Noting that, as $\lambda \rightarrow 0$,

$$
\begin{aligned}
& \Gamma_{0}(\lambda) \sim 1 ; \quad \Gamma_{1}(\lambda) \sim \log (1 / \lambda) \\
& \Gamma_{k}(\lambda) \sim \lambda^{1-k} /(k-1) \quad \text { when } k \geqslant 2,
\end{aligned}
$$

by the standard results for $E_{k}(\lambda)[1$, p. 229] applied to (2.13), we see that, since $x \leqslant x_{0}$, the term in braces in (2.25) is $O(1)$ as $\varepsilon \rightarrow 0$, for all $k \geqslant 1$ (in fact it vanishes as $\varepsilon \rightarrow 0$ when $k=1$ ). Hence, we arrive at the result (2.9). The result (2.10) for the derivative may be proved by analogous reasoning.

Lemma 2 gives precise information about the function $Y(x, \varepsilon)$ both as $\varepsilon \rightarrow 0$ and as $x \rightarrow \infty$. When $x \rightarrow \infty$, it follows, from the standard results for $E_{k}(x)$, that, for each $\varepsilon \in\left(0, \in_{0}\right]$,

$$
Y(x, \varepsilon)=1+O\left(x^{-\alpha k} e^{-x^{\alpha}}\right) .
$$

When $\varepsilon \rightarrow 0$, but $\varepsilon x$ is fixed, with $k \geqslant 2$, we see from (2.9) and (2.11) that

$$
Y(x, \varepsilon) \rightarrow 1 \text { as } \varepsilon \rightarrow 0,
$$

while if $k=1$, this is reduced to $\varepsilon \rightarrow 0$ with $\varepsilon x^{\alpha}$ fixed. These limits, which correspond to the so-called outer limits, may be deduced in the case of the Lagerstrom model problem (when $\alpha=1$ ) on physical grounds.

The following lemma gives an integral estimate for the function $W(x, \varepsilon)$ that will prove very useful in subsequent sections. 
LEMMA 3. Let $W(x, \varepsilon)$ be defined by (2.3), and let $f(1, x)$ satisfy the hypothesis $(F)$. Then,

$$
\varepsilon \int_{1}^{\infty} W(x, \varepsilon) d x=h_{k}(\varepsilon)
$$

where

$$
h_{1}=O(1 / \log (1 / \varepsilon)), \quad h_{2}=O(\varepsilon \log (1 / \varepsilon)), \quad h_{k}=O(\varepsilon), \quad k \geqslant 3
$$

Proof. It may be readily established, by integration-by-parts, that

$$
\int_{1}^{\infty} F_{k}(x, \varepsilon) d x=\left.x F_{k}(x, \varepsilon)\right|_{\infty}+F_{k-1}(1, \varepsilon)-F_{k}(1, \varepsilon),
$$

and it may be deduced from the results of Lemma 2 that $\left.x F_{k}(x, \varepsilon)\right|_{\infty}=0$, for each $\varepsilon \in\left(0, \varepsilon_{0}\right]$. Thus,

$$
\int_{1}^{\infty} W(x, \varepsilon) d x=F_{k-1}(1, \varepsilon) / F_{k}(1, \varepsilon)-1
$$

The results of Lemma 2 now give

$$
F_{k-1}(1, \varepsilon) / F_{k}(1, \varepsilon) \leqslant D(\varepsilon) \Gamma_{k-1}\left(\varepsilon C \alpha^{-1}\right) / \varepsilon \Gamma_{k}\left(\varepsilon C \alpha^{-1}\right),
$$

where $D(\varepsilon)$ is positive, $O(1)$ and nonvanishing as $\varepsilon \rightarrow 0$. The results of the lemma now follow, when we note the asymptotic results (2.26).

COROLlary. Let $f(1, x)$ satisfy the hypothesis $(F)$. Then $Y(x, \varepsilon)$ satisfies the condition

$$
\varepsilon \int_{1}^{\infty}|1-Y(x, \varepsilon)| d x=O(1)
$$

as $\varepsilon \rightarrow 0$.

Proof. From (2.2)

$$
\varepsilon \int_{1}^{\infty}|1-Y(x, \varepsilon)| d x=|a-1| \varepsilon \int_{1}^{\infty} W(x, \varepsilon) d x,
$$

and the result follows from the above lemma.

The fact that $Y(x, \varepsilon)$, the solution of the linear problem $L_{\epsilon}$, satisfies the condition (2.33) leads us to seek solutions $y(x, \varepsilon)$ of the problem $P_{\varepsilon}$ that satisfy the same condition, in the expectation that such solutions might be suitably approximated by $Y(x, \varepsilon)$ as $\varepsilon \rightarrow 0$. This will be seen to be the case in the following sections; but we first consider the assumptions made about the function $f(y, x)$. 
We define the domain $D_{\delta}$, for some $\delta>0$ and independent of $\varepsilon$, by

$$
D_{\delta}=\left\{(x, y, \varepsilon):|1-y| \leqslant|1-a|+\delta, 1 \leqslant x<\infty, 0<\varepsilon \leqslant \varepsilon_{0}\right\}
$$

and make the following assumptions about $f(y, x)$ :

(A1) $f(y, x)$ is Lipschitzian with respect to $y$, uniformly with respect to $x$, with Lipschitz constant $L$ independent of $\varepsilon$; that is,

$$
\left|f\left(y_{1}, x\right)-f\left(y_{2}, x\right)\right| \leqslant L\left|y_{1}-y_{2}\right|
$$

for any $\left(y_{1}, x, \varepsilon\right),\left(y_{2}, x, \varepsilon\right)$ in $D_{\delta}$.

(A2) $f(1, x)$ satisfies the condition $(F)$.

Note from (2.2) that $Y(x, \varepsilon)$ lies in $D_{\delta}$ for any $\delta>0$; and it is this property that motivates the choice of $D_{\delta}$, since we seek solutions $y(x, \varepsilon)$ near $\dot{Y}(x, \varepsilon)$ in some sense. The following lemma gives the properties of solutions that satisfy (2.33) and which lie in $D_{\delta}$ for $f(y, x)$ satisfying the above hypotheses.

LEMMA 4. Let $y(x, \varepsilon)$ be a solution of $P_{\varepsilon}$ in $D_{\delta}$ that satisfies

$$
\varepsilon \int_{1}^{\infty}|1-y(x, \varepsilon)| d x=O(1)
$$

as $\varepsilon \rightarrow 0$. Let $f(y, x)$ satisfy the assumptions $(A 1),(A 2)$. Then, for each $\varepsilon \in\left(0, \varepsilon_{0}\right]$,

and

$$
\begin{gathered}
\min \{a, 1\} \leqslant y(x, \varepsilon) \leqslant \max \{a, 1\}, \\
y^{\prime}(x, \varepsilon) \geqslant 0 \quad \text { if } 0 \leqslant a \leqslant 1,
\end{gathered}
$$

$$
y^{\prime}(x, \varepsilon) \leqslant 0 \text { if } a \geqslant 1 \text {. }
$$

Further, there exist functions $\alpha(x, \varepsilon), \beta(x, \varepsilon)$, continuous and bounded uniformly with respect to $x$ and $\varepsilon$, for $x \in[1, \infty), \varepsilon \in\left(0, \varepsilon_{0}\right]$, such that

and

$$
1-y(x, \varepsilon)=\alpha(x, \varepsilon) W(x, \varepsilon)
$$

$$
y^{\prime}(x, \varepsilon)=\beta(x, \varepsilon) W^{\prime}(x, \varepsilon)
$$

where $W(x, \varepsilon)$ is given by (2.3).

Proof. It is readily shown that for any $(y, x, \varepsilon) \in D_{\delta}$,

$$
x^{-k} \exp \left\{-\varepsilon \int_{1}^{x} f(y, s) d s\right\} \leqslant x^{-k} \exp (\varepsilon L M) \exp \left\{-\varepsilon \int_{1}^{x} f(1, s) d s\right\},
$$

where

$$
M=\int_{1}^{\infty}|1-y(s, \varepsilon)| d s
$$


Further,

$$
x^{-k} \exp \left\{-\varepsilon \int_{1}^{x} f(y, s) d s\right\} \geqslant x^{-k} \exp (-\varepsilon L M) \exp \left\{-\varepsilon \int_{1}^{x} f(1, s) d s\right\}
$$

since, for all $(y, x, \varepsilon) \in D_{\delta}$,

$$
f(y, x) \leqslant f(1, x)+L|1-y| .
$$

Fürthier, since for any $(y, \ddot{x}, \hat{\varepsilon}) \in D_{\delta}$,

$$
f(y, x) \geqslant f(1, x)-L|1-y|,
$$

we see that every solution of $P_{\epsilon}$ satisfying (2.35) may be represented in the form

where

$$
y(x, \varepsilon)=1+(a-1) H_{k}(x, \varepsilon) / H_{k}(1, \varepsilon),
$$

$$
H_{k}(x, \varepsilon)=\int_{x}^{\infty} t^{-k} \exp \left\{-\varepsilon \int_{1}^{t} f(y(s, \varepsilon), s) d s\right\} d t .
$$

The results of the lemma now follow, on applying the results (2.41) and (2.43) to (2.46).

\section{Existence}

In this section, we write the problem $P_{\varepsilon}$ as a pair of integral equations, and apply the contraction mapping principle to this, to deduce the existence of a solution $y(x, \varepsilon)$. The procedure parallels that of [9]. We first write the equation (1.1) in the form

$$
y^{\prime \prime}+\frac{k}{x} y^{\prime}+\varepsilon f(1, x) y^{\prime}=\varepsilon\{f(1, x)-f(y, x)\} y^{\prime} .
$$

Defining the function $v(x, \varepsilon)$ by

$$
v(x, \varepsilon)=1-y(x, \varepsilon),
$$

we may, by constructing the Green's function for the operator on the left-hand side of (3.1), write the problem $\boldsymbol{P}_{\varepsilon}$ as an integral equation for $v$, of the form

$$
v(x, \varepsilon)=(1-a) W(x, \varepsilon)+\varepsilon \int_{1}^{\infty} G(x, s, \varepsilon)\{f(1, x)-f(1-v, x)\} v^{\prime} d s,
$$

where $W(x, \varepsilon)$ is given by (2.3), and the kernel $G(x, s, \varepsilon)$ is given by

$$
G(x, s, \varepsilon)= \begin{cases}\frac{(1-W(s, \varepsilon)) W(x, \varepsilon)}{W^{\prime}(s, \varepsilon)}, & 1 \leqslant s \leqslant x, \\ \frac{W(s, \varepsilon)(1-W(x, \varepsilon))}{W^{\prime}(s, \varepsilon)}, & x \leqslant s \leqslant \infty .\end{cases}
$$


Differentiating (3.3), we obtain

$$
v^{\prime}(x, \varepsilon)=(1-a) W^{\prime}(x, \varepsilon)+\varepsilon \int_{1}^{\infty} G_{x}(x, s, \varepsilon)\{f(1, x)-f(1-v, x)\} v^{\prime} d s .
$$

Defining the functions $\alpha(x, \varepsilon)$ and $\beta(x, \varepsilon)$ by (2.39) and (2.40) respectively, we may write (3.3) and (3.5) as integral equations for $\alpha$ and $\beta$ that take the form

$$
\begin{aligned}
& \alpha(x, \varepsilon)=(1-a)+\varepsilon \int_{1}^{\infty} R(x, s, \varepsilon) H(\alpha, \beta, W) d s, \\
& \beta(x, \varepsilon)=(1-a)+\varepsilon \int_{1}^{\infty} S(x, s, \varepsilon) H(\alpha, \beta, W) d s,
\end{aligned}
$$

where

$$
H(\alpha, \beta, W)=\{f(1, x)-f(1-\alpha W, x)\} \beta .
$$

The kernels $R$ and $S$ are given by

$$
R(x, s, \varepsilon)= \begin{cases}1-W(s, \varepsilon), & 1 \leqslant s \leqslant x, \\ \frac{1-W(x, \varepsilon)}{W(x, \varepsilon)} W(s, \varepsilon), & x \leqslant s \leqslant \infty,\end{cases}
$$

and

respectively.

$$
S(x, s, \varepsilon)= \begin{cases}1-W(s, \varepsilon), & 1 \leqslant s \leqslant x \\ -W(s, \varepsilon), & x \leqslant s<\infty\end{cases}
$$

The following lemma summarizes the manipulations above.

LemMa 5. For each $\varepsilon>0$, every solution $y(x, \varepsilon)$ of $P_{\varepsilon}$ satisfying $(2.35)$, with $f(y, x)$ satisfying $(A 1)$ and $(A 2)$ may be represented in the form (2.39), (2.40), where $\alpha, \beta$ are bounded continuous solutions of the integral equations (3.6), (3.7), whose bounds are $O(1)$ as $\varepsilon \rightarrow 0$.

Conversely, under the assumptions above, the function $y(x, \varepsilon)$ defined by (2.39), (2.40), where $\alpha, \beta$ are bounded continuous solutions of (3.6), (3.7) is a solution of the problem $P_{\varepsilon}$. If these bounds are $O(1)$ as $\varepsilon \rightarrow 0$, this solution satisfies (2.35).

Proof. Obvious, except for the very last part, which is proved by applying Lemma 3.

We now show that the integral equations (3.6), (3.7) have a unique continuous bounded solution $\alpha(x, \varepsilon), \beta(x, \varepsilon)$. We define the set $\Omega$ by

$$
\Omega=\left\{(x, \varepsilon): 1 \leqslant x<\infty, 0<\varepsilon \leqslant \varepsilon_{0}\right\}
$$

for suitably small $\varepsilon_{0}$, and let $C_{M}(\Omega)$ be the set of ordered pairs of functions $(\alpha(x, \varepsilon), \beta(x, \varepsilon))$ that are continuous on $\Omega$ and are uniformly bounded in the sense 
that

$$
|\alpha(x, \varepsilon)| \leqslant M, \quad|\beta(x, \varepsilon)| \leqslant M, \quad \forall(x, \varepsilon) \in \Omega,
$$

where $M$ is a constant, independent of $\varepsilon$. We denote elements of $C_{M}(\Omega)$ by

and define a metric on $C_{M}(\Omega)$ by

$$
\xi=(\alpha, \beta)
$$

$$
\rho_{e}\left(\xi_{1}, \xi_{2}\right)=\max \left\{\sup _{x}\left|\alpha_{1}-\alpha_{2}\right|, \sup _{x}\left|\beta_{1}-\beta_{2}\right|\right\} \text {. }
$$

Then, $C_{M}(\Omega)$ is a complete metric space with respect to $\rho_{\varepsilon}$.

The right-hand sides of the integral equations (3.6), (3.7) define a map $(\alpha, \beta) \rightarrow(\tilde{\alpha}, \widetilde{\beta})$ on $C_{M}(\Omega)$, which we write as

where $T:(\alpha, \beta) \rightarrow(\tilde{\alpha}, \tilde{\beta})$ is given by

$$
\xi=T \xi
$$

$$
\begin{aligned}
& \tilde{\alpha}(x, \varepsilon)=(1-a)+\varepsilon \int_{1}^{\infty} R(x, s, \varepsilon) H(\alpha, \beta, W) d s, \\
& \tilde{\beta}(x, \varepsilon)=(1-a)+\varepsilon \int_{1}^{\infty} S(x, s, \varepsilon) H(\alpha, \beta, W) d s .
\end{aligned}
$$

From (3.15), we have

$$
|\tilde{\alpha}| \leqslant|1-a|+\varepsilon \int_{1}^{\infty}|R||f(1, s)-f(1-\alpha W, s)||\beta| d s .
$$

Noting that $0 \leqslant W \leqslant 1$, with $W(x, \varepsilon)$ monotonically decreasing, while for $M<|1-a|+\delta,(1-\alpha W(s, \varepsilon), s)$ lies in $D_{\delta}$, we may deduce from (3.17) that

$$
|\tilde{\alpha}| \leqslant|1-a|+\varepsilon L M^{2} \int_{1}^{\infty} W(s, \varepsilon) d s .
$$

A similar result holds for the image $\widetilde{\beta}$ of $\beta$, and an application of Lemma 3 gives the following result.

LEMMA 6. The operator $T$ maps $C_{M_{0}}(\Omega)$ into itself, where $M_{0}$ is any value of $M$ which satisfies simultaneously the inequalities

and

$$
|1-a|+h_{k}(\varepsilon) L M^{2} \leqslant M
$$

$$
M<|1-a|+\delta \text {. }
$$

Under the assumptions of Lemma 3 , such an $M$ exists for all $\varepsilon \in\left(0, \varepsilon_{0}\right]$ and $\varepsilon_{0}$ sufficiently small.

Now suppose that

$$
\xi_{1}=T \xi_{1} ; \quad \xi_{2}=T \xi_{2}
$$


From (3.15), we have

$$
\begin{aligned}
\left|\tilde{\alpha}_{1}-\tilde{\alpha}_{2}\right| & \leqslant \varepsilon \int_{1}^{\infty}|R|\left|H\left(\alpha_{1}, \beta_{1}, W\right)-H\left(\alpha_{2}, \beta_{2}, W\right)\right| d s \\
& \leqslant 2 M(1+L) \rho_{\varepsilon}\left(\xi_{1}, \xi_{2}\right) \varepsilon \int_{1}^{\infty} W d s .
\end{aligned}
$$

Noting a similar inequality for $\left|\widetilde{\beta}_{1}-\widetilde{\beta}_{2}\right|$, we obtain

$$
\rho_{\epsilon}\left(\xi_{1}, \xi_{2}\right) \leqslant 2 M(1+L) h_{k}(\varepsilon) \rho_{e}\left(\xi_{1}, \xi_{2}\right),
$$

on applying Lemma 3. The following result follows.

LEMMA 7. The operator $T$ is a contraction on $C_{M_{0}}(\Omega)$ when

$$
M_{0}<\left(2(1+L) h_{k}(\varepsilon)\right)^{-1} \text {. }
$$

Clearly, (3.19), (3.20) and (3.24) may be satisfied for all $\varepsilon \in\left(0, \varepsilon_{0}\right]$ and $\varepsilon_{0}$ small enough. We thus have our basic existence result for this problem.

THEOREM 1. For all $\varepsilon \in\left(0, \varepsilon_{0}\right]$ and $\varepsilon_{0}$ sufficiently small, the map $T$ has a unique fixed point in the space $C_{M_{0}}(\Omega)$ when $M_{0}$ satisfies (3.19) and (3.20).

Proof. A direct application of the contraction mapping principle [7, p. 27). Lemma 5 then gives the result below.

THEOREM 2. Let $f(y, x)$ satisfy the conditions $(A 1),(A 2)$. Then, for all $\varepsilon \in\left(0, \varepsilon_{0}\right]$ and $\varepsilon_{0}$ sufficiently small, $P_{\epsilon}$ has a unique solution $y(x, \varepsilon)$ satisfying the condition (2.35).

It would appear that to remove the condition (2.35) on solutions of $P_{\epsilon}$ would prove very difficult without further assumptions about the behaviour of the function $f(y, x)$. However, in at least one instance, namely that of the Lagerstrom model problem, it may be proved [9] that all solutions of the problem have a representation of the form (2.39), (2.40), and hence all satisfy (2.35). Thus, in this instance, the restricted uniqueness of Theorem 2 becomes a global uniqueness result.

We also note that the results of Theorem 2 will hold if we replace the Lipschitz condition of (A1) by one of the form

$$
\left|f\left(y_{1}, x\right)-f\left(y_{2}, x\right)\right| \leqslant L\left|y_{1}-y_{2}\right|^{p}, \quad p \geqslant 1 .
$$

Since $0 \leqslant W^{p} \leqslant W \leqslant 1$, the inequality (3.19) would be replaced by

$$
1+h_{k}(\varepsilon) M^{1+p} \leqslant M
$$


which could be satisfied for small $\varepsilon$. It is doubtful if the results would hold for $0<p<1$, however.

Finally, it is worth noting that, for solutions of $P_{\varepsilon}$ satisfying (2.35), we in fact have, by Lemma 3 ,

$$
\varepsilon \int_{1}^{\infty}|1-y(x, \varepsilon)| d x \rightarrow 0 \text { as } \varepsilon \rightarrow 0
$$

\section{Asymptotic expansions}

Resulting from the contraction mapping principle, we have the existence of an iterative scheme

$$
\xi_{n}=T \xi_{n-1}, \quad n=1,2, \ldots,
$$

which converges to the fixed point $\xi$ of the map $T$ in $C_{M_{0}}(\Omega)$, for any initial iterate $\xi_{0}$ in this space. Repeating the analysis set out in [9], and choosing $\left(\alpha_{0}, \beta_{0}\right)=(0,0)$ as our initial iterate, we obtain the result

$$
\begin{aligned}
& \alpha(x, \varepsilon)=(1-a)+O\left(h_{k}\right), \\
& \beta(x, \varepsilon)=(1-a)+O\left(h_{k}\right),
\end{aligned}
$$

for $(\alpha, \beta)$, the fixed point of the map $T$. Noting $(2.39),(2.40)$ we then have the following theorem.

THEOREM 3. Under the assumptions of Theorem 2, the solution $Y(x, \varepsilon)$ of $L_{\epsilon}$ is an asymptotic approximation as $\varepsilon \rightarrow 0$ to the solution $y(x, \varepsilon)$ of $P_{\varepsilon}$, with

$$
y(x, \varepsilon) \sim Y(x, \varepsilon) ; \quad y^{\prime}(x, \varepsilon) \sim Y^{\prime}(x, \varepsilon)
$$

uniformly with respect to $x \in[1, \infty)$.

Again, repeating the analysis of [9], we obtain the result below.

THEOREM 4. Under the assumption of Theorem 2 , the solution to $P_{6}$ has a generalized asymptotic expansion

$$
\begin{aligned}
& y(x, \varepsilon)=Y(x, \varepsilon)+\sum_{n=1}^{N} \nu_{n}(\varepsilon) Y_{n}(x, \varepsilon)+O\left(v_{N+1}(\varepsilon)\right) \\
& y^{\prime}(x, \varepsilon)=Y^{\prime}(x, \varepsilon)+\sum_{n=1}^{N} \nu_{n}(\varepsilon) Y_{n}(x, \varepsilon)+O\left(\nu_{N+1}(\varepsilon)\right)
\end{aligned}
$$

with

$$
\nu_{n}(\varepsilon)=h_{k}(\varepsilon)^{n}
$$


and

$$
\begin{aligned}
& Y_{n}(x, \varepsilon)=-W(x, \varepsilon) \phi_{n}(x, \varepsilon), \\
& Y_{n}(x, \varepsilon)=-W^{\prime}(x, \varepsilon) \psi_{n}(x, \varepsilon) .
\end{aligned}
$$

The functions $\phi_{n}(x, \varepsilon)$ and $\psi_{n}(x, \varepsilon)$ are $O(1)$ as $\varepsilon \rightarrow 0$, uniformly with respect to $x \in[1, \infty)$.

The iterative scheme (4.1) may be used to calculate the expansion (5.5), through the relationship (2.39). Thus, a uniformly valid first approximation to $y(x, \varepsilon)$ is given by (4.1) and (3.15) as

where

$$
y(x, \varepsilon)=1+\alpha_{2}(x, \varepsilon) W(x, \varepsilon)+O\left(h_{k}(\varepsilon)^{2}\right),
$$

$$
\alpha_{2}(x, \varepsilon)=(1-a)+\varepsilon \int_{1}^{\infty} R(x, s, \varepsilon) H(1,1, W) d s
$$

and

$$
H(1,1, W)=f(1, x)-f(1-W, x) .
$$

Provided the integration involved in (4.11) can be carried out, this procedure yields the uniformly valid asymptotic approximation to order $h_{k}^{2}$. In principle, the method is much simpler than the method of matched expansions, and any difficulty can be seen to lie in the form of the function $f(y, x)$. In the particular case of the Lagerstrom model problem $(a=0, f(y, x)=y)$, this calculation may be carried out with relative ease (Shepherd [10]) to yield, after one integration, a composite expansion that is asymptotically equivalent to that obtained by matching techniques after several matching processes [3]. In this sense, then, the process above is the more efficient of the two.

\section{Conclusion}

The procedures set out in this paper will allow us to establish desirable properties of nonlinear singular perturbation problems $P_{\varepsilon}$, providing we can pick the "right" linear problem $L_{\epsilon}$. The particular linearization (1.4) chosen here is suitable because of the weak nonlinearity in (1.1). Removal of this type of nonlinearity poses serious questions about the choice of the linear problem $L_{\varepsilon}$; for example, if we consider the Lagerstrom model for compressible low Reynolds number flow,

$$
y^{\prime \prime}+\frac{k}{x} y^{\prime}+y^{\prime 2}+\varepsilon y y^{\prime}=0, \quad x \in(1, \infty),
$$

with the same boundary conditions, (1.4) is not a suitable linear problem $L_{\varepsilon}$. Further, it is difficult to construct such a linear problem for (5.1), on an ad hoc basis. 
This question has received considerable attention in a recent paper by Rosenblat [8], and later by Shepherd [11], in which the authors consider nonlinear singular perturbation problems $P_{\epsilon}$ on a finite interval $[0,1]$, with the usual "boundary layer" at $x=0$. An equivalent linear problem $L_{\epsilon}$ is proposed, with undetermined coefficients that satisfy specific conditions, and the problem $L_{\varepsilon}$ is actually constructed by the requirement that its solution provide a suitable approximation to that of $\boldsymbol{P}_{\boldsymbol{\epsilon}}$.

\section{Acknowitedgement}

The author wishes to thank Professor Simon Rosenblat of the University of Melbourne for his help during the solution of this problem.

\section{REFERENCES}

[1] M. Abramowitz and I. A. Stegun, Handbook of mathematical functions (National Bureau of Standards, Washington, D.C., 1965).

[2] W. B. Bush, "On the Lagerstrom mathematical model for viscous flow at low Reynolds number", S.I.A.M. J. Appl. Math. 20 (1971), 279-287.

[3] J. D. Cole, Perturbation methods in applied mathematics (Blaisdell, New York, 1968).

[4] G. C. Hsiao, "Singular perturbations for a nonlinear differential equation with a small parameter", S.I.A.M. J. Math. Anal. 4 (1973), 283-301.

[5] P. A. Lagerstrom, Asymptotic methods for the study of the Navier-Stokes equations (Calif. Instit. Tech. Translation Project, Pasadena, Calif., 1965).

[6] P. A. Lagerstrom and R. Casten, "Basic concepts underlying singular perturbation techniques", S.I.A.M. Review 14 (1972), 63-120.

[7] L. Liusternik and V. Sobolev, Elements of functional analysis (Ungar, 1965).

[8] S. Rosenblat, "Asymptotically equivalent singular perturbation problems", Stud. Appl. Math. 55 (1976), 249-280.

[9] S. Rosenblat and J. Shepherd, "On the asymptotic solution of the Lagerstrom model equation", S.I.A.M. J. Appl. Math. 29 (1975), 110-120.

[10] J. J. Shepherd, Singular perturbation problems (M.Sc. Thesis, University of Melbourne, 1975).

[11] J. J. Shepherd, Nonlinear singular perturbation problems (Ph.D. Thesis, University of Melbourne, 1977).

Department of Mathematics

University of Western Australia

Nedlands, W.A. 6009

Australia 